\title{
Depressive mood, bonding failure, and abusive parenting among mothers with three-month-old babies in a Japanese community
}

\author{
Toshinori Kitamura $^{1,2}$, Yukiko Ohashi ${ }^{1}$, Sachiko Kita ${ }^{1,3}$, Megumi Haruna ${ }^{3}$, Reiko Kubo ${ }^{4}$ \\ ${ }^{1}$ Kitamura Institute of Mental Health, Tokyo, Japan \\ ${ }^{2}$ Department of Psychiatry, Graduate School of Medicine, Nagoya University, Nagoya, Japan \\ ${ }^{3}$ Department of Midwifery and Women's Health, University of Tokyo, Tokyo, Japan \\ ${ }^{4}$ Kumamoto Prefecture, Kumamoto, Japan \\ Email: kitamura@kokoroclinic.jp
}

Received 13 April 2013; revised 16 May 2013; accepted 24 May 2013

Copyright (C) 2013 Toshinori Kitamura et al. This is an open access article distributed under the Creative Commons Attribution License, which permits unrestricted use, distribution, and reproduction in any medium, provided the original work is properly cited.

\begin{abstract}
Objective: To investigate the relationships between depressive mood, bonding failure, and abusive parenting. Method: We distributed questionnaires to 1198 mothers attending a three-month postnatal health check-up in rural areas in Japan. The questionnaires assessed these three variables along with demographics. We evaluated the causal relationships by comparing different structural equation models to the data. Results: Although all the models fit the data well, the best Akaike Information Criterion was obtained from a model where both depressive mood and bonding failure predict abusive parenting, but depressive mood and bonding failure do not predict each other directly. The determinant coefficient of child abuse in the final model was 0.13 . Discussion: We found depressive mood and bonding failure during postpartum period impacted negatively on mother's parenting behaviour. Because only $13 \%$ of the variance of abusive parenting was explainable by this model, further study should be needed to identify other risk factors of child abuse. Similarly, midwives and public health nurse in community should pay attention to not only depression but also bonding failure to prevent child abuse.
\end{abstract}

Keywords: Postnatal Depression; Bonding Failure; Child Abuse; Mothers of Neonates; Structural Equation Analysis

\section{INTRODUCTION}

Childbirth is a great event in family life. The first rela-

${ }^{*}$ Conflict of Interest: None. tionship in a child's life is with his or her mother. Consequently, when discussing mental health issues during the postnatal period, not only is the mother's psychological adjustment such as postnatal depression important, but also her caring style (particularly child abuse) and attitude (particularly maternal bonding) towards her newborn. Hence, depression, bonding failure, and abusive parenting are three topics related to mental health during the postnatal period.

Depression is a major health issue during the perinatal period. O'Hara and Swain [1] have reported that the prevalence of depression in the postnatal period is around $13 \%$ in western countries [2]. A multicenter longitudinal study has indicated the prevalence of postnatal depression in Japan is 5\% [3]. In addition to depression causing distress for mothers, it may also affect the cognitive and emotional development of children [4].

Recently, dysfunction in the mother-infant relationship has been reported as another important perinatal mental health issue [5]. Some mothers experience a feeling of rejection or even hatred towards their child. A disturbed mother-infant relationship is termed as bonding failure or bonding disorder. Although depression and bonding failure often coexist, they are believed to be two separate issues. The etiology has not been thoroughly examined [6]. However, Brockington [5] has noted that bonding failure may differ from depression, and listed unwanted pregnancy as a candidate correlate.

A third perinatal mental health issue is abusive child rearing. Child abuse has been a hot topic of family studies. The prevalence of physical and psychological child abuse in Japan is as high as that reported in Western countries [7,8]. Abusive rearing begins when the child is an infant [9]. Mothers frequently yell at or refuse to talk 
to their babies. These behaviors are frequently observed among Japanese mothers of infants [10].

The associations of these three aforementioned issues are unclear. Depressed mothers may be more likely to behave abusively towards their babies. Mothers who abuse their infants may feel guilty, and thus become depressed. Alternatively, those who feel hostile towards their infants may feel self-reproaching and depressed. However, the causal relationships between these three variables (depression, bonding failure, and child abuse) have not been examined.

Recent years have seen an increasing concern on the mental health of childbearing women in Japan. In 2006, the Ministry of Health, Labour, and Welfare issued a report "Healthy Parents and Children 21" [11] that paced reduction of postnatal depression and reduction of child death by abuse as two of the main goals of the government. However, it seems without sufficient evidence that local governments tied the postnatal depression and child abuse. For example, three Prefectures (Miyagi, Fukushima, and Nagano) and two cities (Nerima, Suginami and Fukuyama) adopted the Edinburgh Postnatal Depression Scale, a screening instrument of postnatal depression, as a means to detect mothers with potential of child abuse [12]. They encourage public health nurses to identify mothers with postnatal depression in order to initiate "early intervention of child abuse". This may be immature as a community health policy making. Moreover, if the link between depression and child abuse were not the case, such policy may lead to unnecessary stigma toward postnatal depression. This may in turn inhibit formal support seeking among mothers with postnatal depression.

Although a longitudinal follow-up study of a large population may be most suitable to study these causal relationships, studies with a longitudinal design are expensive. A more affordable preliminary approach is a cross-sectional study to examine the relationships between the three variables using a variety of path models. Path models allow researchers to posit causality from one variable to another. Structured equation modeling with goodness-of-fit indices such as Akaike Information Criterion (AIC) enables researchers to compare path models in terms of how well the models fit the data. Hence, such a comparison of models may lead to a better cue to presume the time sequence of the three.

\section{METHOD}

\subsection{Participants}

We solicited all mothers attending three-month postnatal health check-ups in 4 cities, 21 towns, and 5 villages in the Kumamoto Prefecture (located in the center of the Kyushu Island southwest of Tokyo) to participate in this questionnaire survey. The survey was anonymous. Par- ticipants were asked to complete the questionnaire while waiting for their check-ups and to return it prior to leaving. Of the 1405 questionnaires distributed, 1293 (92\%) were returned.

\subsection{Instruments}

Due to time restrictions and physical limitations (mothers completed the questionnaire while holding their babies), a single page questionnaire was designed. This resulted in reducing the number of items as much as possible.

\subsubsection{Depressive Mood}

Depressive mood during the postnatal period was assessed by a single item: "Have you felt sad or miserable during the last week?" The question was assessed on a four-point scale from "never" (0) to "almost always" (3). A single item is not a very accurate measure of depression. A scale with multiple items would be more desirable. However, we believe that this single-item assessment is sufficient in a community-based study because other studies have reported that such a single item is a valid screening instrument of depression $[13,14]$.

\subsubsection{Bonding Failure}

Two items from the Mother-Infant Bonding Questionnaire (MIBQ) were extracted: "I feel protective towards my baby" and "I feel close to my baby" (reverse items). The MIBQ aims to elicit mothers' feelings of rejection, alienation, and neutrality towards their infants. Good test-retest reliability and construct validity have been reported [15]. Moreover, its validity has also been reported $[16,17]$. In this study, we used the Japanese version [18].

\subsubsection{Child Abuse}

Mothers' abusive parenting was rated by three items extracted from the Child Form of the Conflict Tactic Scale (CTS) [19]. These questions inquire about the number of occurrences of abusive behaviors since childbirth. They included; \#1 "insulted or swore at [the baby]"; \#2 "sulked or refused to talk about an issue"; and \#3 "cried". They were selected because these questions scored the highest in a previous study on Japanese mothers' child abuse of one-month-old babies [10].

\subsubsection{Demographic Features}

We asked about the ages of the mother and her partner as well as the infants' birth weights and gender.

\subsection{Ethics}

This research project was approved by the Ethical Committee of Kumamoto University Graduate School of Life Sciences. 


\subsection{Statistical Analyses}

All the statistical analyses were conducted using the Statistical Package for Social Science (SPSS) version 19.0 and Amos 19.0. After examining the means, SDs, and skewness of all the variables used in this study, we logarithmically transformed the variables with a skewness greater than two. For a bird's eye view of the data, a correlation matrix of all the variables was obtained. From here, we compared different models with Depression, Bonding Failure, and Child Abuse as predictors of each other using structured equation modeling (SEM).

The fit of the model with the data was examined in terms of chi-squared (CMIN), goodness-of-fit index (GFI), adjusted goodness-of-fit index (AGFI), comparative fit index (CFI), and root mean square error of approximation (RMSEA). According to conventional criteria, a good fit would be indicated by $\mathrm{CMIN} / \mathrm{df}<2$, GFI $>$
0.95, AGFI $>0.90, \mathrm{CFI}>0.97$, and RMSEA $<0.05$, while $\mathrm{CMIN} / \mathrm{df}<3$, GFI $>0.90$, AGFI $>0.85$, CFI $>$ 0.95 , and RMSEA $<0.08$ demonstrate an acceptable fit [20]. To compare all the models and to determine the best model to fit the data, we used the Akaike Information Criterion (AIC). An AIC model more two point higher is regarded as superior to another [21].

\section{RESULTS}

Of the 1293 mothers returning the questionnaire, 1198 (93\%) were fully completed with the necessary data for all the variables used in this study. Table 1 lists the mean, $\mathrm{SD}$, range, and skewness of all the variables. Because the scores of Depressive Mood, the two Bonding Failure, and three Child Abuse items exceeded 2.0 and were positively skewed, they were logarithmically transformed. This transformation reduced their skewness, but Abuse

Table 1. Descriptive properties of variables used in this study $(\mathrm{N}=1198)$.

\begin{tabular}{|c|c|c|c|c|c|c|}
\hline & Mean & SD & Minimum & Maximum & Skewness & $\begin{array}{l}\text { Skewness after } \\
\text { Logarithmic } \\
\text { Transformation }\end{array}$ \\
\hline Depression & 0.31 & 0.58 & 0 & 3 & 2.00 & 1.42 \\
\hline Bonding Failure \#1 & 1.2 & 0.4 & 1 & 3 & 2.29 & 2.08 \\
\hline Bonding Failure \#2 & 1.1 & 0.4 & 1 & 3 & 3.21 & 2.84 \\
\hline Child Abuse \#1 & 0.1 & 0.6 & 0 & 10 & 8.93 & 6.01 \\
\hline Child Abuse \#2 & 0.6 & 1.9 & 0 & 30 & 8.10 & 2.57 \\
\hline Child Abuse \#3 & 0.3 & 1.2 & 0 & 15 & 5.95 & 3.29 \\
\hline Own Age & 29.3 & 5.1 & 16 & 45 & 0.18 & - \\
\hline Partner's Age & 31.2 & 6.2 & 17 & 59 & 0.61 & - \\
\hline Number of Children & 1.8 & 0.9 & 1 & 7 & 1.16 & - \\
\hline Birth Weight & 3074 & 415 & 1310 & 4756 & -0.17 & - \\
\hline Gender of Baby (Boy 1; Girl 2) & 1.50 & 0.50 & 1 & 2 & 0.01 & - \\
\hline
\end{tabular}

Bonding Failure \#1 is "I feel protective towards my baby"; \#2 is "I feel close to my baby"; Child Abuse \#1 is "insulterd or sware (at the baby)"; \#2 is "sulked or refused to talk about an issue"; \#3 is "cried".

Table 2. Correlates of all the variables used in this study.

\begin{tabular}{|c|c|c|c|c|c|c|c|c|c|c|}
\hline & 1 & 2 & 3 & 4 & 5 & 6 & 7 & 8 & 9 & 10 \\
\hline 1. Depressive $\operatorname{mood}^{\mathrm{a}}$ & - & & & & & & & & & \\
\hline 2. Bonding Failure $\# 1^{\mathrm{a}}$ & $0.12^{* * *}$ & - & & & & & & & & \\
\hline 3. Bonding Failure $\# 2^{\mathrm{a}}$ & $0.12^{* * *}$ & $0.59^{* * *}$ & - & & & & & & & \\
\hline 4. Child Abuse $\# 1^{\mathrm{a}}$ & $0.11^{* * *}$ & 0.04 & $0.09^{* *}$ & - & & & & & & \\
\hline 5. Child Abuse $\# 2^{\mathrm{a}}$ & $0.21^{* * *}$ & $0.09^{* *}$ & $0.10^{* *}$ & $0.25^{* * *}$ & - & & & & & \\
\hline 6. Child Abuse $\# 3^{\mathrm{a}}$ & $-0.12^{* * *}$ & 0.03 & 0.05 & $0.31^{* * *}$ & $0.30^{* * *}$ & - & & & & \\
\hline 7. Own age & $-0.07^{*}$ & -0.03 & -0.01 & -0.01 & $-0.07^{*}$ & $-0.12^{* * *}$ & - & & & \\
\hline 8. Partner's age & $-0.08^{* *}$ & -0.05 & -0.03 & -0.02 & -0.05 & $-0.11^{* * *}$ & $0.74^{* * *}$ & - & & \\
\hline 9. Number of children & -0.04 & 0.00 & 0.00 & -0.02 & $-0.12^{* * *}$ & $-0.10^{* *}$ & $0.36^{* * *}$ & $0.31^{* * *}$ & - & \\
\hline 10. Birth weight & 0.02 & 0.04 & 0.01 & -0.05 & -0.06 & 0.03 & $0.06^{*}$ & 0.04 & $0.12^{* * *}$ & - \\
\hline 11. Gender of baby & -0.04 & 0.01 & 0.02 & 0.01 & -0.05 & -0.02 & 0.03 & 0.03 & $0.07^{*}$ & $-0.06^{*}$ \\
\hline
\end{tabular}

${ }^{\mathrm{a}}$ Logarithmically transformed; ${ }^{*} p<0.05 ;{ }^{* *} p<0.01 ;{ }^{* * *} p<0.001$. 
item \#1 was still very positively skewed (6.01).

Table 2 depicts the correlations between all the variables. Depressive mood was significantly correlated with Bonding Failure and Child Abuse items as well as younger ages of the mother and her partner. The two Bonding Failure items were correlated with each other. Similarly, the Child Abuse items were correlated. In addition, the Child Abuse items were generally correlated with Bonding Failure items, younger age of the couple, and fewer numbers of children. As expected, younger couples were more likely to have fewer children.

In the SEM models (Figure 1), we excluded Child Abuse item \#1 due to its high skewness even after the logarithmic transformation. Additionally, we excluded birth weight and gender of the baby because they were not significantly correlated with Depression, Bonding Failure, or Child Abuse items. In these models, we set

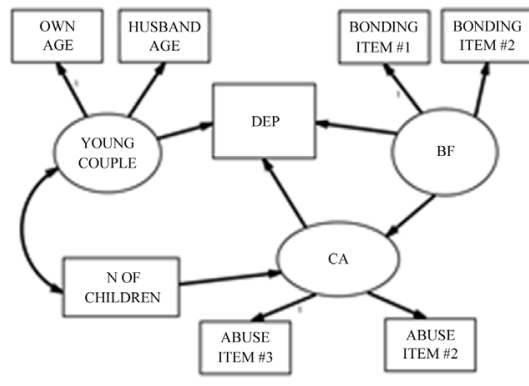

(A) $\mathrm{BF} \rightarrow \mathrm{CA} \rightarrow \mathrm{DEP}$

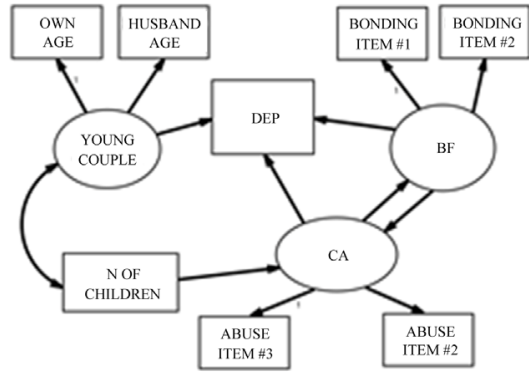

(D) $\mathrm{BF} \gtrless \mathrm{CA} \rightarrow \mathrm{DEP}$

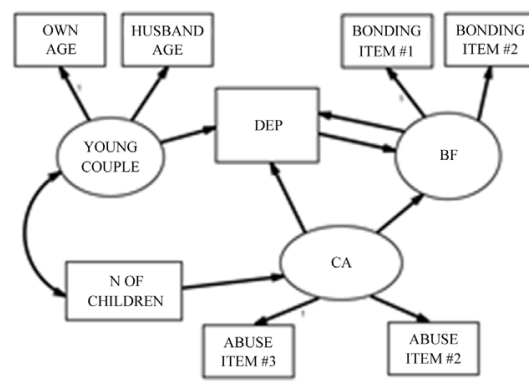

(G) $\mathrm{CA} \rightarrow \mathrm{BF} \rightleftarrows \mathrm{DEP}$

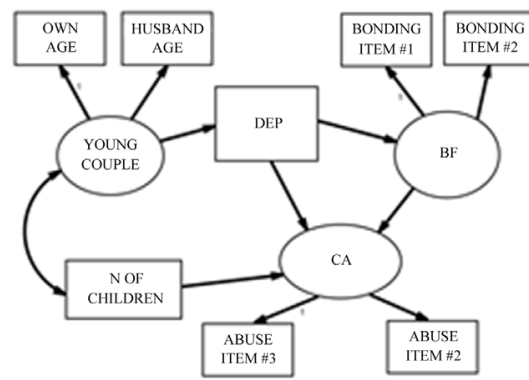

(J) $\mathrm{DEP} \rightarrow \mathrm{BF} \rightarrow \mathrm{CA}$

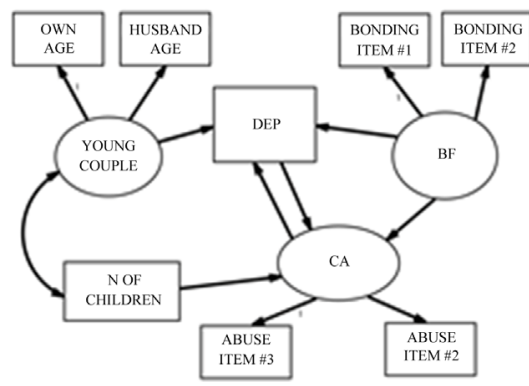

(B) $\mathrm{BF} \rightarrow \mathrm{CA} \leftrightarrows \mathrm{DEP}$

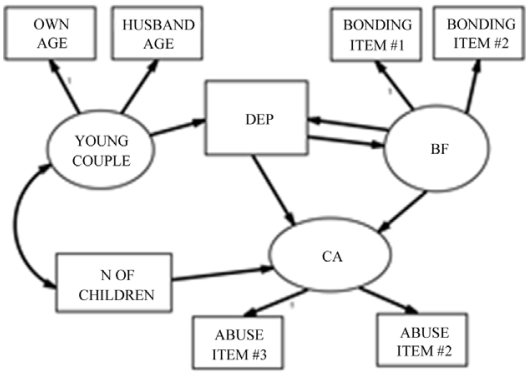

(E) $\mathrm{BF} \rightleftarrows \mathrm{DEP} \rightarrow \mathrm{CA}$

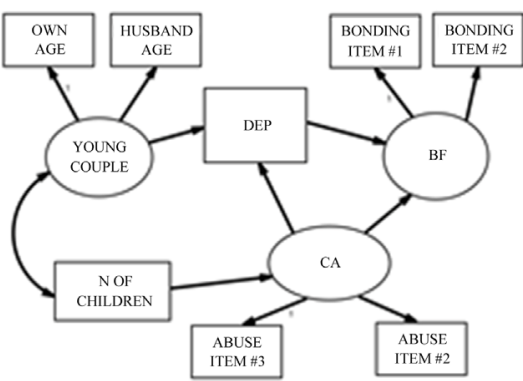

(H) $\mathrm{CA} \rightarrow \mathrm{DEP} \rightarrow \mathrm{BF}$

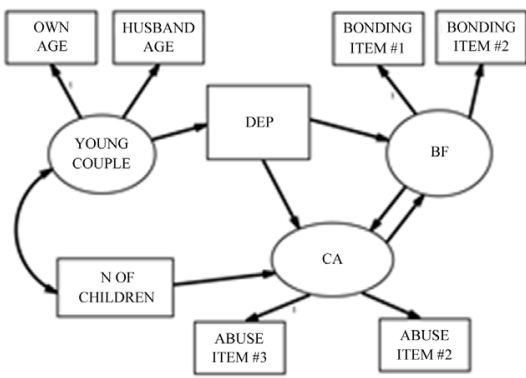

(K) $\mathrm{DEP} \rightarrow \mathrm{BF} \rightleftarrows \mathrm{CA}$

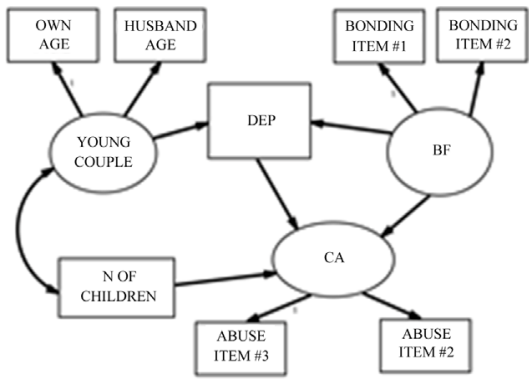

(C) $\mathrm{BF} \rightarrow \mathrm{DEP} \rightarrow \mathrm{CA}$

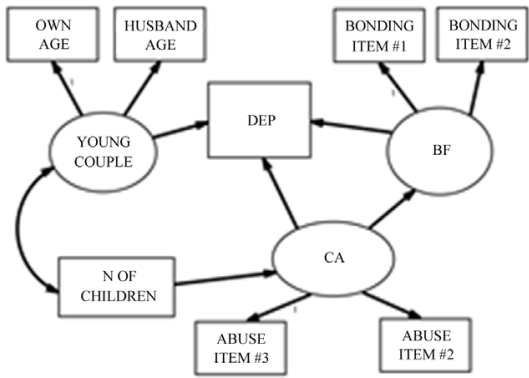

(F) $\mathrm{CA} \rightarrow \mathrm{BF} \rightarrow \mathrm{DEP}$

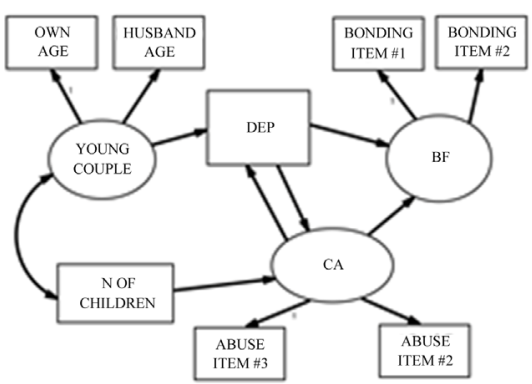

(I) $\mathrm{CA} \rightleftarrows \mathrm{DEP} \rightarrow \mathrm{BF}$

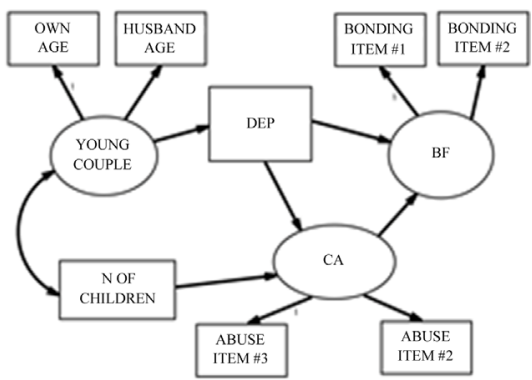

(L) $\mathrm{DEP} \rightarrow \mathrm{CA} \rightarrow \mathrm{BF}$

Figure 1. Multiple SEM models of the associations between Bonding Failure (BF), Depression (DEP), and Child Abuse (CA). 
up three latent variables: Young Couple (influenced by the ages of the mother and her partner), Bonding Failure (influenced by the two logarithmically transformed MIBS items), and Child Abuse (predicted by items 2 and 3 of the CTS, which were logarithmically transformed). As described above, Depressive Mood was rated by the single item.

Then we posited that 1) Young Couple would predict Depression; 2) the Number of Children would predict Child Abuse; and 3) Young Couple and Number of Children would co-vary. Between Depressive Mood, Bonding Failure, and Child Abuse, we set up 12 prediction models, which followed one of three types of patterns. In one type of pattern, one variable predicted another either directly or via a mediating variable (patterns A, C, F, H, $\mathrm{J}$, and $\mathrm{L}$ ). In the second type of pattern, one variable predicted two variables, which also predicted each other (patterns B, G, and K). In the last pattern, two variables predicted each other and these two also predicted the third variable (patterns D, E, and I).

All the models fit the data well: GFI $>0.996$, AGFI $>$ 0.990 , CFI $>0.997$, and RMSEA $<0.015$ (Table 3). However, Model E yielded the best AIC score as it was more than 2.0 lower than models A, C, D, F, G, H, J, and

Table 3. Comparison of SEM models. BF, Bonding Failure; CA, Child Abusive; DEP, Depressive Mood; AIC difference is greater than two higher in \# than $\$$.

\begin{tabular}{|c|c|c|c|c|c|c|c|c|}
\hline Model & Content & CMIN & $\mathrm{df}$ & GFI & AGFI & CFI & RMSEA & AIC \\
\hline A & $\mathrm{BF} \rightarrow \mathrm{CA} \rightarrow \mathrm{DEP}$ & 13.920 & 13 & 0.997 & 0.992 & 0.999 & 0.008 & $59.920 \$$ \\
\hline $\mathrm{B}$ & $\mathrm{BF} \rightarrow \mathrm{CA} \leftrightarrows \mathrm{DEP}$ & 16.650 & 15 & 0.997 & 0.992 & 0.999 & 0.010 & 58.650 \\
\hline $\mathrm{C}$ & $\mathrm{BF} \rightarrow \mathrm{DEP} \rightarrow \mathrm{CA}$ & 13.920 & 13 & 0.997 & 0.992 & 0.999 & 0.008 & $59.920 \$$ \\
\hline $\mathrm{D}$ & $\mathrm{BF} \rightleftarrows \mathrm{CA} \rightarrow \mathrm{DEP}$ & 19.074 & 15 & 0.996 & 0.991 & 0.998 & 0.015 & $61.074 \$$ \\
\hline $\mathrm{E}$ & $\mathrm{BF} \rightleftarrows \mathrm{DEP} \rightarrow \mathrm{CA}$ & 15.706 & 15 & 0.997 & 0.992 & 1.000 & 0.006 & $57.706 \#$ \\
\hline $\mathrm{F}$ & $\mathrm{CA} \rightarrow \mathrm{BF} \rightarrow \mathrm{DEP}$ & 15.199 & 13 & 0.997 & 0.991 & 0.999 & 0.012 & $61.199 \$$ \\
\hline G & $\mathrm{CA} \rightarrow \mathrm{BF} \rightleftarrows \mathrm{DEP}$ & 19.862 & 15 & 0.996 & 0.990 & 0.997 & 0.016 & $61.862 \$$ \\
\hline $\mathrm{H}$ & $\mathrm{CA} \rightarrow \mathrm{DEP} \rightarrow \mathrm{BF}$ & 14.915 & 13 & 0.997 & 0.992 & 0.999 & 0.011 & $60.915 \$$ \\
\hline I & $\mathrm{CA} \rightleftarrows \mathrm{DEP} \rightarrow \mathrm{BF}$ & 16.936 & 15 & 0.997 & 0.992 & 0.999 & 0.010 & 58.936 \\
\hline $\mathrm{J}$ & $\mathrm{DEP} \rightarrow \mathrm{BF} \rightarrow \mathrm{CA}$ & 13.958 & 13 & 0.997 & 0.992 & 0.999 & 0.008 & $59.958 \$$ \\
\hline $\mathrm{K}$ & $\mathrm{DEP} \rightarrow \mathrm{BF} \rightleftarrows \mathrm{CA}$ & 16.022 & 15 & 0.997 & 0.992 & 0.999 & 0.008 & 58.022 \\
\hline $\mathrm{L}$ & $\mathrm{DEP} \rightarrow \mathrm{CA} \rightarrow \mathrm{BF}$ & 14.915 & 13 & 0.997 & 0.992 & 0.999 & 0.011 & $60.915 \$$ \\
\hline
\end{tabular}


L. Models K and B exhibited the second best fit, and Model I exhibited the fourth best fit.

In Model E (Figure 2), Young Couple predicted Depressive Mood significantly. Additionally, a significant inverse prediction occurred from the Number of Children to Child Abuse. Both Depressive Mood and Bonding Failure predicted Child Abuse. However, Depressive Mood and Bonding Failure could not predict directly each other.

\section{DISCUSSION}

The present study aims to elucidate the causal relationships between three variables: depressive mood, bonding failure, and abusive parenting. Although a longitudinal follow-up observational study would be ideal, due to financial constraints, we adopted a cross-sectional design of a large sample in which we compared different causality models in terms of their fit to the data. This study demonstrates that depressive mood, bonding failure, and abusive parenting are weakly correlated with one other. Younger ages of the mother and her partner are correlated with depressive mood. The number of children is inversely correlated with abusive parenting.

The best-fit model (Model E) indicates that both depresssive mood and bonding failure would significantly predict abusive parenting. This model shows superiority to eight other models in terms of AIC scores, but its superiority is not substantial compared to three models (Models K, $\mathrm{B}$, and I). Model $\mathrm{K}$ also suggests a causal path from depressive mood to abusive parenting. Although Models B and I set bilateral paths between depression and abusive parenting, neither of the two paths is significant. The path from depressive mood to abusive parenting barely reaches a significant level in Model I. Hence, we hypothesize that a path from depressive mood towards abusive

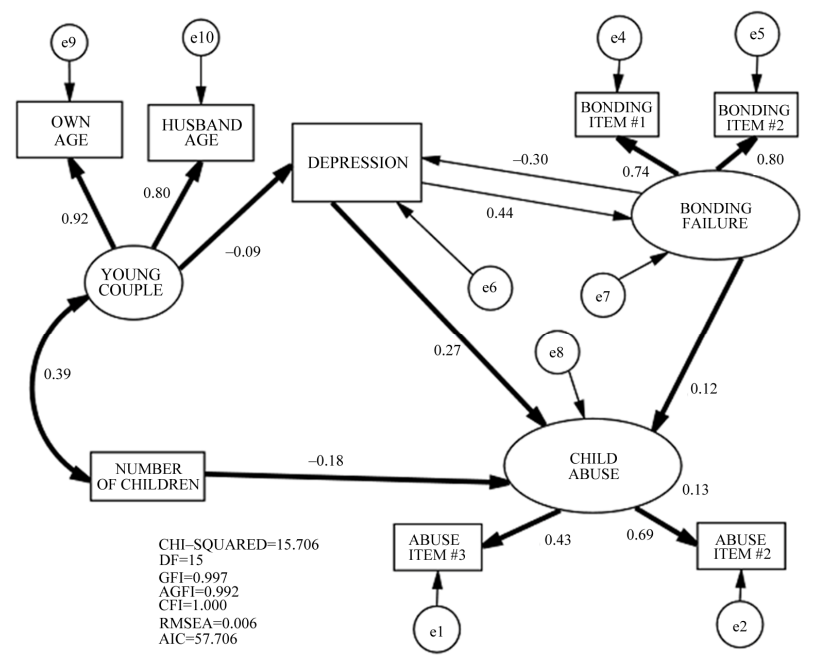

Figure 2. Final SEM model of the association between Bondeing Failure, Depression, and Child Abuse. (Significant paths and covariances are in bold.) parenting is very possible.

The path from bonding failure towards abusive parenting is significant in both Models E and B. Although Model I only posits a unilateral path from abusive parenting towards bonding failure, this path is significant. However, when a bilateral path is set between bonding failure and abusive parenting in Model $\mathrm{K}$, the path from abusive parenting towards bonding failure loses its significance. Hence, we posit that a path from bonding failure towards abusive parenting is possible, but that the reverse may also be true.

Finally, unilateral paths from depressive mood towards bonding failure and vice versa are all significant in Models $\mathrm{K}, \mathrm{B}$, and I, but these paths lose significance when a bilateral path is posited in Model E. Considering these observations, it is possible that both depressive mood and bonding failure could lead to abusive parenting, but depressive mood and bonding failure might be independent.

In addition, young age of the couples is a risk factor for depressive mood, while a fewer number of children is a risk factor for abusive parenting. Therefore, young couples with one baby may be at a greater risk of abusive parenting than older couples or couples with multiple children.

Another important feature of this study is the determinant coefficient of Child Abuse is only 0.13. This means that more than $80 \%$ of the variability of abusive parenting is something other than depression and bonding failure, suggesting it would be unwise to identify cases of depression in mothers of infants as a preventive means of child abuse in a community as advocated by local governments in Japan. Instead mental health professionals should use an instrument to measure abusive parenting directly in setting up community health service systems.

Limitations of this study should be considered before concluding our paper. First, the cross-sectional nature of this study strongly warns against making immature conclusions. Non-recursive models such as the ones used in this study assume an equilibrium where the system is in a steady sate [21]. This means that any changes, which underlie the feedback between more than one variable, have manifested their effects in the model. We simply claim that this study may cast light on the possibility of causal relationships between depressive mood, bonding failure, and child abuse. However, this study is unique in that it includes a fairly large sample using SEM. Further multi-wave studies may be promising to clarify this temporal relationship.

Second, all three variables in study were measured by a small number of items, which may be the cause of unreliability. Additionally, we relied solely on the mothers' own reports, which may be a source of bias (shared observer bias). The third drawback is the scarcity of other potential causes of child abuse. We only studied birth weight and gender of the baby. Studies with a full list of 
possible risk factors may cast a different light on the results.

Considering these drawbacks, this study suggests a possible path from depressive mood and bonding failure towards abusive parenting. Further longitudinal studies are necessary to clarify this possibility.

\section{REFERENCES}

[1] O'Hara, M.W. (2009) Postpartum depression: What we know. Journal of Clinical Psychology, 65, 1258-1269. doi:10.1002/jclp.20644

[2] O'Hara, M.W. and Swain, A.M. (1996) Rate and risk of postpartum depression: A meta-analysis. International Review of Psychiatry, 8, 37-54. doi:10.3109/09540269609037816

[3] Kitamura, T., Yoshida, K., Okano, T., Kinoshita, K., Hayashi, M., Toyoda, N., et al. (2006) Multicentre prospective study of perinatal depression in Japan: Incidence and correlates. Archives of Women's Mental Health, 9, 121130. doi:10.1007/s00737-006-0122-3

[4] Beck, C.T. (1995) The effects of postpartum depression on maternal-infant interaction: A metal-analysis. Nursing Research, 44, 298-304. doi:10.1097/00006199-199509000-00007

[5] Brockington, I. (2004) Diagnosis and management of postpartum disorders: A review. World Psychiatry, 3, 89-95.

[6] Kumar, R.C. (1997) "Anybody's child”: Severe disorders of mother-to-infant bonding. The British Journal of Psychiatry, 171, 175-181. doi:10.1192/bjp.171.2.175

[7] Kitamura, T., Kitahara, T., Koizumi, T., Takashi, N., Chiou, M.L. and Fujihara, S. (1995) Epidemiology of child abuse in Japan: How big is the iceberg? Journal of Forensic Psychiatry, 6, 425-431.

[8] Kitamura, T., Kaibori, Y., Takara, N., Oga, H., Yamauchi, K. and Fujihara, S. (2000) Child abuse, other early experiences and depression: I. Epidemiology of parental loss, child abuse, perceived rearing experience and early life events among a Japanese community population. Archives of Women's Mental Health, 3, 47-52.

[9] Wu, S.S., Ma, C., Carter, R.L., Ariet, M., Feaver, E.A. and Resnick, M.B., et al. (2004) Risk factors for infant maltreatment: A population-based study. Child Abuse Neglect, 28, 1253-1264. doi:10.1016/j.chiabu.2004.07.005

[10] Kitamura, T., Takauma, F., Tada, K., Yoshida, K. and
Nakano, H. (2004) Postnatal depression, social support, and child abuse. World Psychiatry, 3, 100-101.

[11] Yamagata, Z. (2001) Healthy parents and children 21. http://rhino.med.yamanashi.ac.jp/sukoyaka/abstract.html

[12] Japan Association of Public Health Center Directors (2012) Japan association of public health center directors. http://www.phcd.jp

[13] Chochinov, H.M., Wilson, K.G., Enns, M. and Lander, S. (1997) Are you depressed? Screening for depression in the terminally ill. American Journal of Psychiatry, 154, 674-676.

[14] Mitchell, A.J. and Coyne, J.C. (2007) Do ultra-short screening instruments accurately detect depression in primary care? A pooled analysis and meta-analysis of 22 studies. British Journal of General Practice, 57, 144-151.

[15] Taylor, A., Atkins, R., Kumar, R., Adams, D. and Glover, V. (2005) A new mother-infant bonding scale: Links with early maternal mood. Archives of Women's Mental Health, 8, 45-51. doi:10.1007/s00737-005-0074-Z

[16] Figueiredo, B., Costa, R., Pacheco, A. and Pais, A. (2009) Mother-to-infant emotional involvement at birth. Maternal and Child Health Journal, 13, 539-549. doi:10.1007/s10995-008-0312-x

[17] Wittkowski, A., Wiek, A. and Mann, S. (2007) An evaluation of two bonding questionnaires: A comparison of the mother-to-infant bonding scale with the postpartum bonding questionnaire in a sample of primiparous mothers. Archives of Women's Mental Health, 10, 171-175. doi:10.1007/s00737-007-0191-y

[18] Yoshida, K., Yamashita, H., Conroy, S., Marks, M. and Kumar, C. (2012) A Japanese version of mother-to-infant bonding scale: Factor structure, longitudinal changes and links with maternal mood during the early postnatal period in Japanese moters. Archives of Women's Mental Health, 15, 343-352.

[19] Straus, M.A. (1979) Measuring intrafamilial conflict and violence: Conflict tactics scales. Journal of Marriage and Family, 41, 75-88.

[20] Schermelleh-Engel1, K., Moosbrugger, H. and Müller, H. (2003) Evaluating the fit of structural equation models: Tests of significance and descriptive goodness-of-fit measures. Methods of Psychological Research Online, 8, 2374.

[21] Kline, R.B. (2005) Principles and practice of structural equation modeling. 2nd Edition, Guilford Press, New York. 